\title{
Clinical Safety and Efficacy of Autologous Bone Marrow-Derived Mesenchymal Stem Cell Transplantation in Sensorineural Hearing Loss Patients
}

\author{
Ho Seok Lee ${ }^{1}$, Woo Jin Kim², Ji Sun Gong ${ }^{1}$, and Kyoung Ho Park ${ }^{1}$ \\ ${ }^{1}$ Department of Otorhinolaryngology-Head and Neck Surgery, The Catholic University of Korea College of Medicine, Seoul, \\ ${ }^{2}$ Department of Otorhinolaryngology, Yonsei University College of Medicine, Seoul, Korea
}

\author{
Received June 8, 2017 \\ Revised July 17, 2017 \\ Accepted August 3, 2017
}

Stem cell transplantation represents a promising therapy for several degenerating and necrotic diseases. In several animal studies, we could find hearing restoration after inoculation of the mesenchymal stem cells' as well as mesenchymal stem cells' differentiation of hair cells and spiral ganglion. But until now, no clinical study has been reported directly for the human being. In this pilot studies, we applied mesenchymal stem cells to human beings trans-venously. Although we verified the safety of the autologous bone marrow stem cell transplantation in sensorineural hearing loss patients but we could not achieve significant improvement in hearing.

J Audiol Otol 2018;22(2):105-109 Electrical stimulation.

\section{Introduction}

Hearing loss is a severe disorder affecting communication and quality of life. Numerous studies have demonstrated a consistently negative impact of hearing loss on communication and in addition, it has recently been shown to be a risk factor for dementia [1]. Some authors maintain that it is associated with increasing mortality [2].

Sensorineural hearing loss, damage of cochlear sensory cells or neuron is the most common reason among them. It is estimated that the number of patients with neuron-related hearing loss will double by the year 2030 [3]. Commonly, sensorineural hearing loss is caused by genetic dysfunction, ototoxic drugs, noise [4].

The current therapeutic options for patients with sensorineural hearing include hearing amplification and cochlear implantation. While these interventions are effective in many

This is an Open Access article distributed under the terms of the Creative Commons Attribution Non-Commercial License (http://creativecommons.org/licenses/by-nc/4.0/) which permits unrestricted non-commercial use, distribution, and reproduction in any medium, provided the original work is properly cited. cases, some patients still struggle with the physiologic and psychosocial impacts of deafness.

Recently, transplantation of stem cells has emerged as a promising therapeutic option for the treatment of intractable hearing loss [5]. Hearing is mediated by mechano-sensory hair cells located within the inner ear cochlea. In mammal, mechano-sensory hair cells in the cochlea are only generated during a short period of embryonic development. Thus, the lack of cochlear regenerative potential draws permanence of hearing loss [6]. Recently, many studies revealed that mesenchymal stem cells from umbilical cord blood (UCB) or Bone marrow (BM) can differentiate into multiple lineage cells. In spite of mesenchymal stem cell (MSC) transplantation has not been tried for hearing loss yet, MSCs have been applied to many other organ disorders like hematological disorders, cardiovascular disease, osteogenesis imperfecta, some neurological pathologies and even cancer. According to the myocardial infarction, some articles revealed MSC transplantation has more benefit in increasing left ventricular ejection fraction and systolic wall motion [7].

Previously we revealed the UCB-derived mesenchymal stro- 
mal cells can affect to recover hearing from mice as well as they can differentiate into auditory hair cells and neuronal cells in vitro [3]. From these research results, we tried transplantation of MSCs in patients with sensorineural hearing loss.

\section{Case Report}

Patients are asked for their informed consent prior to entering research studies.

We conducted two pilot clinical trials for patients with sensorineural hearing loss one with and one without auditory neuropathy. Auditory neuropathy is a problem in cochlea auditory nerve or spiral ganglion cells might be better recovery from mesenchymal stem cell transplantation. This study protocol and procedures were reviewed and approved by the Ministry of Food and Drug Safety (KFDA) of Korea and the Institutional Review Board (KIRB-00414-199) at the School of Medicine, Catholic University of Korea.

\section{Case 1}

A 67-year-old female was a consulted patient from neurosurgery department for hearing impairment after retro-sigmoid craniotomy with microvascular decompression for the periorbital spasm. Her pure tone thresholds were out of scale on left and $15 \mathrm{~dB}$ on right. Auditory brainstem response (ABR) thresholds were also out of scale on left and $30 \mathrm{~dB}$ on right. Safety assessment was also checked before the surgery. Her hematology, biochemistry, coagulation, and urine tests were all within the normal range. Several treatment options were suggested to the patient and she finally agreed with BM derived mesenchymal stem cell injection.
For collection of autologous BM derived mesenchymal stem cell, we aspirated BM of $10 \mathrm{~mL}$ from iliac crest under local anesthesia. Promptly, it is transported to the manufacturer for isolation and culture of MSC (Pharmicell Co Ltd.). After four weeks, we could culture autologous MSCs of 50 million (MSC4: $5 \times 10^{7}$ cell $/ 10 \mathrm{~mL}$ ).

Before transplantation, we secured subclavian line for the safe infusion. Under general anesthesia, the patient was lied in prone position. BM derived stem cells were mixed with the normal saline and infused intravenously through subclavian line slowly (60 mL/hr, for $20 \mathrm{~min})$. For the effective migration of the stem cell (homing to the cochlea), we applied electrical stimulation of $1.5 \mathrm{~mA}$ to the promontory continuously before and after the stem cell infusion. Antero-inferior incision was made on the left tympanic membrane (experimental ear). Then nerve stimulator was located at the promontory. After infusion, incision margin was everted with the pick and paper patch was applied. She discharged without any audio-vestibular or systemic complication. She was followed up as one month interval (Fig. 1).

As for results, we could not find any complication and side effect associated with stem cell transplantation. There were also no changes in laboratory findings, hematology, biochemistry, coagulation (Fig. 2). But we could not have any change of hearing [subjective symptom, pure tone audiometry (PTA), oto-acoustic emission (OAE) and ABR] during 12 months.

After 3 years of infusion, there was no any problem with stem cell therapy.

\section{Case 2}

A 55-year-old male patient came to clinic, complaining of
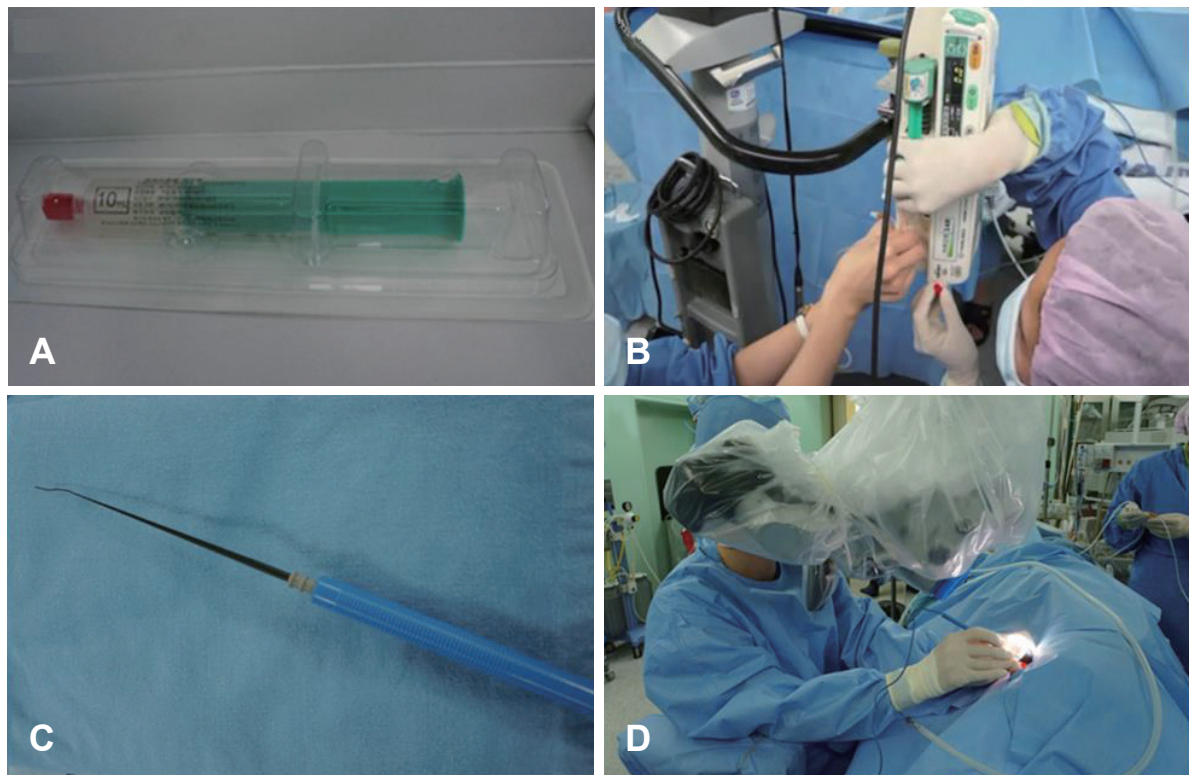

Fig. 1. BM derived stem cells were mixed with the normal saline and infused intravenously through subclavian line slowly. For the effective migration of the stem cell (homing to the cochlea), we applied electrical stimulation to the promontory continuously before and after the stem cell infusion. A: Autologous MSCs of 50 million (MSC4: $5 \times 10^{7}$ cell $/ 10 \mathrm{~mL}$ ). B: $\mathrm{BM}$ derived stem cells were mixed with the normal saline and infused intravenously through subclavian line slowly (60 mL/hr, for $20 \mathrm{~min}$ ). C, D: For the effective migration of the stem cell, electrical sti-mulation of $1.5 \mathrm{~mA}$ was applied to the promontory continuously before and after the stem cell infusion. BM: bone marrow, MSC: mesenchymal stem cell. 
Fig. 2. The audiograms of Case 1 patient (pure tone audiogram, otoacoustic emission, auditory brainstem response) before (A) and after (B) treatment. The patient did not showed any improvement in hearing tests.
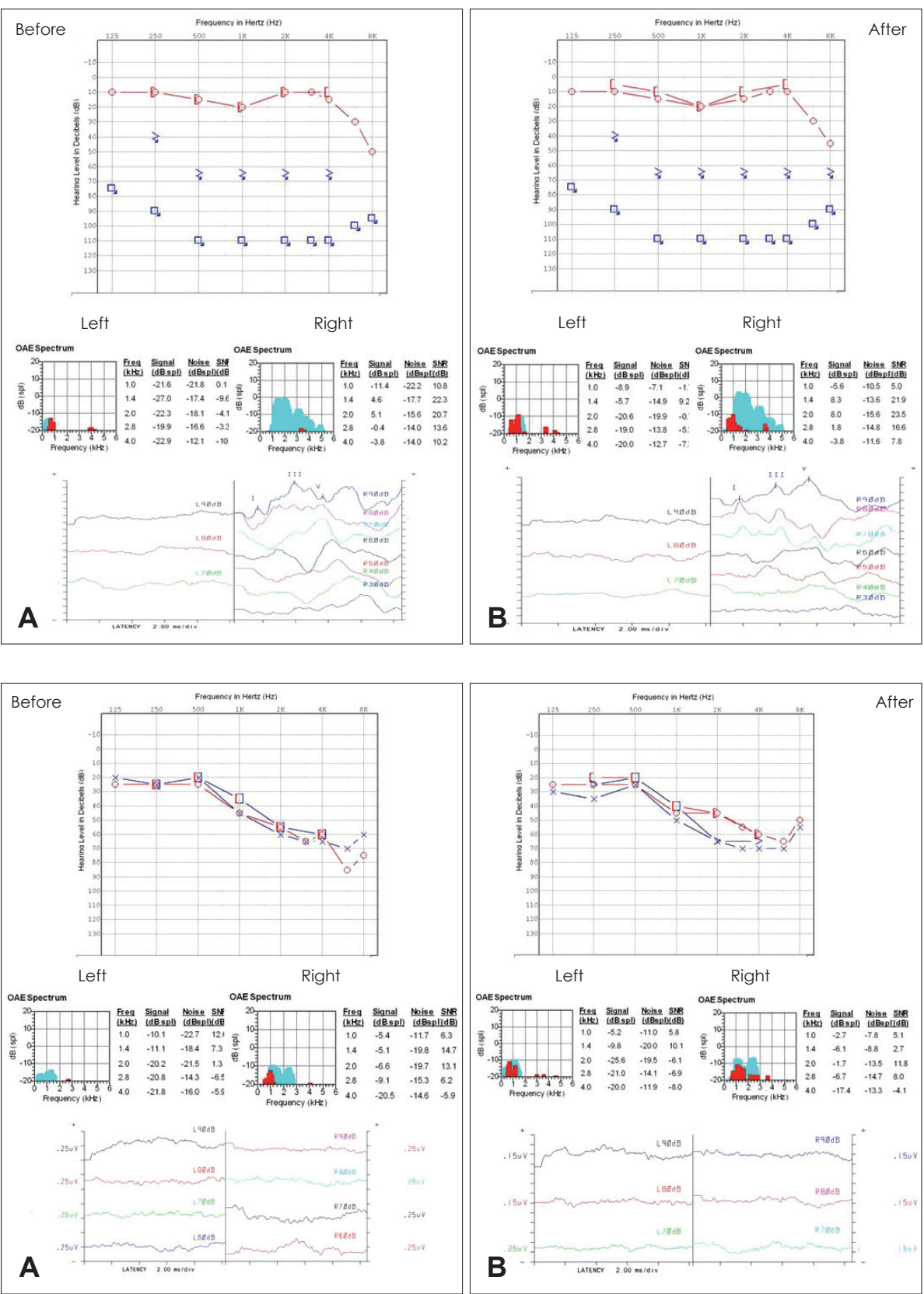

Fig. 3. The audiograms of Case 2 patient (pure tone audiogram, otoacoustic emission, auditory brainstem response) before (A) and after (B) treatment. The patient did not showed any improvement in hearing tests. cell therapy.

\section{Discussion}

Stem cell transplantation represents a promising therapy for several degenerating and necrotic diseases. Restoration of spiral ganglion neurons and hair cells are the targets for stem cell therapy in the treatment of hearing loss [8]. Differentiation of implanted MSCs to fibrocytes is critical for the efficacy of the transplantation treatment. Fibrocytes, particularly in the mesenchymal non-sensory regions, play an important role in cochlear physiology. Fibrocytes constitute a significant After 3 years of infusion, there was no problem with stem 
proportion of the cells in the cochlea, and five types of fibrocytes are recognized based on morphological and cyto-chemical characteristics [9]. Fibrocytes in the spiral ligament (SL) of the cochlea are connected with gap junctions and play key roles in potassium uptake from the perilymph and recycling of those ions back to endolymph. In aging human with hearing difficulty, fibrocytes are progressively lost from the SL of the cochlea, suggesting the importance of fibrocytes for auditory capacity [10]. Some animal studies revealed that MSCs can differentiate to the hair cells and spiral ganglion, and by auditory function test, hearing can be restored after inoculation. Furthermore, no reports revealed significant complications [11]. Therefore, we hypothesized that hearing loss can be restored in sensorineural hearing loss if we can transplant MSCs to the cochlea safely in human.

Cell delivery to the cochlea is one the crucial mechanisms to establish an efficient stem cell therapy in patients with hearing impairment. It can either be done by intravenous administration or by local transplantation. For local administration, cells can be infused in the round window, scala tympani or scala media, or direction injection into the modiolus. Kasagi, et al. studied with mice and they infused MSCs via microtube, set on the holes, both lateral and posterior semicircular canal via temporal bone drilling. All of them show positive effects on hearing loss in previous animal studies [11,12]. However, possible damage to the cochlea still exists by direct or local inoculation.

For intravenous administration, the volume of MSCs is need to be increased, because the stem cells are barely bypassing the blood brain barrier. In our previous mice study, we injected MSCs via the brachial vein using a Hamilton syringe (Supelco, Bellefonte, PA, USA). As mentioned before, there are various inoculation methods reported by animal studies and none of them reported serious complications. But we applied MSCs intravenously due to the possibility of changes in component and volume of endolymph if we inoculate MSCs directly. Changed components and volume may lead to vertigo and tinnitus which cannot be checked by animal study. We also concerned about the environment the MSCs grow. The components of endolymph are not suitable to cells live. In our previous experiment (not published yet), we identified $0.1 \%$ of MSCs in endolymph when administered via intraperitoneally or intravenously in previous animal study.

Recently, the factors which can draw implanted stem cells to the target organ is widely studied, and Kamiya, et al. [13] maintained that transplanted the MSCs to the lateral semicircular canal after the induction of stem cell homing factors (stromal cell-derived factor 1 alpha; Monocyte chemoattractant protein-1) in the host cochlear tissue, and their receptors in transplanted MSCs can make better results. Although there were many animal studies have been done before, there were no prospective, statistically validated, long term outcome studies about the stem cell therapy on the patients with hearing loss.

We applied electrical stimuli $1.2-1.5 \mathrm{~mA}$ on the promontory during infusion of MSCs for the homing effect. Electrical stimulation is a well established clinical tool that has beneficial applications in neuromodulation, cardiac pacing, cardiac differentiation of human stem cells and brain stimulation [14]. We hypothesized that the clinical utility of electrical stimulation may expand to include the directed recruitment and migration of transplanted MSCs.

In our previous animal study with mice, we observed both restoration of hearing by ABR threshold, distortion product oto-acoustic emission level and increase of spiral ganglion at all turns of the cochlea by human amniotic membrane derived MSCs and UCB-MSC regardless of possibility of immunologic reaction owing to the xenotransplantation.

The BM derived MSCs are known to be one of the most promising stem cell sources for cell replacement therapy because they can differentiate into neurons and can be used by the patient's own bone. Furthermore, Cho, et al. [12] revealed MSCs from BM has effective in restoration of hearing in mice study. In these clinical trials, we must consider immunologic problems with transplantation in human clinical trials. So we administered autologous BM derived MSCs. Although we could not get the effective and promising audiological results, there was no any side effect or complications associated stem cell therapy. We assumed that autologous BM derived MSC transplantation in sensorineural hearing loss (SNHL) patients is safe treatment modality. But it does not mean that MSC transplantation itself is absolutely safe modality in treating SNHL because we have lack of long term follow up result yet. It may have a potential to make some changes to human body including cancer.

In conclusion, during this trial, we verified the safety of the autologous BM stem cell treatment in sensorineural hearing loss patients. But we could not achieve significant improvement in hearing. Even though we could not identify the hearing gain in this trial, the autologous BM stem cell transportation is still promising by improvement of the transportation method and adequate patient selection in the area of regeneration medicine.

\section{Acknowledgments}

This research was supported by the national strategic center for clinical research of Korea and was supported by the Research Fund of the Catholic University ENT Alumni (KC13CNSF0282). 


\section{Conflicts of interest}

The authors have no financial conflicts of interest.

\section{REFERENCES}

1) Gordon-Salant S. Hearing loss and aging: new research findings and clinical implications. J Rehabil Res Dev 2005;42(4 Suppl 2):9-24.

2) Appollonio I, Carabellese C, Frattola L, Trabucchi M. Effects of sensory aids on the quality of life and mortality of elderly people: a multivariate analysis. Age Ageing 1996;25:89-96.

3) Kil K, Choi MY, Kong JS, Kim WJ, Park KH. Regenerative efficacy of mesenchymal stromal cells from human placenta in sensorineural hearing loss. Int J Pediatr Otorhinolaryngol 2016;91:72-81.

4) Guo W, Yuan Y, Hou Z, Wang X, Yang S. Profiles of the auditory epithelia related microRNA expression in neonatal and adult rats. Eur J Med Res 2014;19:48.

5) Suzuka Y, Schuknecht HF. Retrograde cochlear neuronal degeneration in human subjects. Acta Otolaryngol Suppl 1988;450:1-20.

6) Sinkkonen ST, Chai R, Jan TA, Hartman BH, Laske RD, Gahlen F, et al. Intrinsic regenerative potential of murine cochlear supporting cells. Sci Rep 2011;1:26.

7) Giordano A, Galderisi U, Marino IR. From the laboratory bench to the patient's bedside: an update on clinical trials with mesenchymal stem cells. J Cell Physiol 2007;211:27-35.
8) Tamura T, Nakagawa T, Iguchi F, Tateya I, Endo T, Kim TS, et al. Transplantation of neural stem cells into the modiolus of mouse cochleae injured by cisplatin. Acta Otolaryngol Suppl 2004:65-8.

9) Spicer SS, Schulte BA. Differentiation of inner ear fibrocytes according to their ion transport related activity. Hear Res 1991;56:53-64.

10) Adams JC. Immunocytochemical traits of type IV fibrocytes and their possible relations to cochlear function and pathology. J Assoc Res Otolaryngol 2009;10:369-82.

11) Kasagi H, Kuhara T, Okada H, Sueyoshi N, Kurihara H. Mesenchymal stem cell transplantation to the mouse cochlea as a treatment for childhood sensorineural hearing loss. Int J Pediatr Otorhinolaryngol 2013;77:936-42.

12) Cho YB, Cho HH, Jang S, Jeong HS, Park JS. Transplantation of neural differentiated human mesenchymal stem cells into the cochlea of an auditory-neuropathy guinea pig model. J Korean Med Sci 2011; 26:492-8

13) Kamiya K, Yum SW, Kurebayashi N, Muraki M, Ogawa K, Karasawa $\mathrm{K}$, et al. Assembly of the cochlear gap junction macromolecular complex requires connexin 26. J Clin Invest 2014;124:1598-607.

14) Hernández D, Millard R, Sivakumaran P, Wong RC, Crombie DE, Hewitt AW, et al. Electrical stimulation promotes cardiac differentiation of human induced pluripotent stem cells. Stem Cells Int 2016; 2016:1718041 